\title{
Distinctive spatiotemporal expression patterns for neurotrophins develop in gustatory papillae and lingual tissues in embryonic tongue organ cultures
}

Received: 23 March 2000 / Accepted: 25 July 2000 / Published online: 21 November 2000

(C) Springer-Verlag 2000

\begin{abstract}
Brain-derived neurotrophic factor (BDNF) and neurotrophin-3 (NT-3) mRNAs are expressed in the developing rat tongue and taste organs in specific spatiotemporal patterns. BDNF mRNA is present in the early lingual gustatory papilla epithelium, from which taste buds eventually arise, prior to the arrival of gustatory nerve fibers at the epithelium, whereas NT-3 initially distributes in the mesenchyme. However, a direct test for neural dependence of neurotrophin expression on the presence of innervation in tongue has not been made, nor is it known whether the patterns of neurotrophin expression can be replicated in an in vitro system. Therefore, we used a tongue organ culture model that supports taste papilla formation while eliminating the influence from sensory nerve fibers, to study neurotrophin mRNAs in lingual tissues. Rat tongue cultures were begun at embryonic day 13 or 14 (E13, E14), and BDNF, NT-3, nerve growth factor (NGF) and neurotrophin-4 (NT-4) mRNAs were studied at $0,2,3$ and 6 days in culture. BDNF transcripts were localized in the gustatory epithelium of both developing fungiform and circumvallate papillae after 2 or 3 days in culture, and NT-3 transcripts were in the subepithelial mesenchyme. The neurotrophin distributions were comparable to those in vivo at
\end{abstract}

This research was supported by the School of Dentistry, University of Michigan, the Karolinska Institute, the Swedish Medical Association, the David and Astrid Hageléns Foundation, and the National Institute on Deafness and Other Communication Disorders, NIH grant DC00456 to C.M.M.

C.A. Nosrat (

Division of Molecular Neurobiology, Department of Neuroscience, Karolinska Institutet, 171 77, Stockholm, Sweden

e-mail: nosrat@umich.edu

Tel.: +1-734-615-4388, Fax: +1-734-647-2110

D.K. MacCallum

Department of Cell and Developmental Biology, Medical School, University of Michigan, Ann Arbor, USA

C.A. Nosrat · C.M. Mistretta

Department of Biologic and Materials Sciences,

School of Dentistry, University of Michigan, Ann Arbor, MI 48109-1078, USA
E13-E16. In 6-day tongue cultures, however, BDNF transcripts in anterior tongue were not restricted to fungiform papillae but were more widespread in the lingual epithelium, while the circumvallate trench epithelium exhibited restricted BDNF labeling. The NT-3 expression pattern shifted in 6-day organ cultures in a manner comparable to that in the embryo in vivo, and was expressed in the lingual epithelium as well as mesenchyme. NGF mRNA expression was subepithelial throughout 6 days in cultures. NT- 4 mRNA was not detected. The neurotrophin mRNA distributions demonstrate that temporospatial localization of neurotrophins observed during development in vivo is retained in the embryonic tongue organ culture system. Furthermore, initial neurotrophin expression in the developing lingual epithelium, mesenchyme, and/or taste papillae is not dependent on intact sensory innervation. We suggest that patterns of lingual neurotrophin mRNA expression are controlled by the influence of local tissue interactions within the tongue at early developmental stages. However, the eventual loss of restricted BDNF mRNA localization from fungiform papillae in anterior tongue suggests that sensory innervation may be important for restricting the localized expression of neurotrophins at later developmental stages, and for maintaining the unique phenotypes of gustatory papillae.

Keywords Neurotrophins - Gustation · Brain-derived neurotrophic factor - Neurotrophin-3 - Organ culture . In situ hybridization $\cdot$ Rat (Sprague Dawley)

\section{Introduction}

Brain-derived neurotrophic factor (BDNF) is localized within the epithelium of embryonic rat tongue regions that develop into gustatory papillae, and the neurotrophin remains within differentiating papillae at subsequent stages of morphogenesis (Nosrat 1998; Nosrat et al. 1996; Nosrat and Olson 1995). Furthermore, once papillae have formed, BDNF is expressed in specific pa- 
pilla regions where taste buds will eventually develop. Thus, this neurotrophin is an important and specific early marker for presumptive and early taste epithelium, in both rats and humans (Nosrat and Olson 1995; Nosrat et al. 2000). Neurotrophin-3 (NT-3), in contrast, is initially localized in mesenchymal tissues of the developing tongue, and subsequently shifts to include epithelial distributions in lingual regions that will acquire tactile sensation (Nosrat et al. 1996; Nosrat and Olson 1998). Using a culture system of embryonic tongue that supports formation and morphogenesis of the gustatory papillae in a patterned array (Mbiene et al. 1997), we now demonstrate that BDNF and other neurotrophins are expressed in vitro, in tissue distributions that are similar to those observed during development in vivo. Further, the in vitro system demonstrates that these neurotrophin expression patterns emerge in the absence of an intact sensory innervation to the tongue. However, after several days in culture, some loss of BDNF patterning takes place whereas other neurotrophin patterns are sustained.

The lingual papillae that support taste buds are the fungiform, circumvallate, and foliate (Mistretta 1991). These gustatory organs are distributed in a specific pattern on the tongue (Mistretta 1972). Other nongustatory epithelial specializations are the filiform papillae, cornified spines that are distributed over the anterior twothirds of the tongue and in more plate-like organs on the intermolar eminence.

On the rat tongue at embryonic day 13 (E13) the lingual epithelium is histologically homogeneous over the lingual swellings of mesenchyme (Mbiene et al. 1997). By E14 the tongue has assumed its spatulate form and the first signs of epithelial eminences that will differentiate into fungiform and circumvallate papillae are observed. Between E14 and E15 the fungiform and circumvallate papillae become distinct, and morphogenesis continues through gestation. Taste buds, however, do not appear histologically in rat gustatory papillae until E20, and thus, taste bud development is essentially a postnatal event (Mistretta 1972).

Lingual innervation from the combined lingual/chorda tympani nerve (anterior tongue) and glossopharyngeal nerve (posterior tongue) is not within the tongue core until E14 and approaches the epithelium at E15 (Mbiene and Mistretta 1997; Rochlin and Farbman 1998). In vitro systems of tongue organ (Mbiene et al. 1997) or papilla (Farbman and Mbiene 1991) cultures have demonstrated that papillae develop without an intact sensory innervation. Indeed, from organ cultures it is clear that fungiform papillae develop in their stereotypic distribution in rows on the anterior tongue and that the circumvallate papilla develops in the central midline location, at appropriate embryonic stages, without intact sensory nerves (Mbiene et al. 1997).

We hypothesized that BDNF and NT-3 mRNA expression is not dependent on an intact sensory innervation for a restricted and organ-specific distribution during tongue development. Tongue organ cultures were initiated at E13, before the tongue is fully formed, and E14, when the fungiform papillae are beginning to differentiate, to study papilla development and neurotrophin mRNA expression in vitro. Expression of BDNF, NT-3, NGF, and NT-4 mRNA was compared following various times in culture, and between cultured tongues and previous in utero developmental stages.

\section{Materials and methods}

Timed, pregnant Sprague-Dawley rats were obtained from Charles River Breeders. The day on which the rats were sperm-positive (a detectable sperm plug) was designated as embryonic day 0 (E0). Pregnant rats at E13 or E14 were anesthetized with an intraperitoneal injection of sodium pentobarbital $(50 \mathrm{mg} / \mathrm{kg}$ body weight). Anesthetized embryos were removed under aseptic procedures, and placed in a sterile Petri dish containing Earl's balanced salt solution (EBSS), buffered with $20 \mathrm{~m} M$ HEPES (pH 7.4), and gentamicin sulfate $(20 \mu \mathrm{g} / \mathrm{ml})$. The embryos were decapitated, and the heads were moved to fresh EBSS. Tongues (E13 and E14) or tongue with a portion of mandible (E13) were dissected.

Tongue organ cultures. Procedures for organ culture technique have been described previously (Mbiene et al. 1997). Briefly, tongues were oriented with dorsal surface upward on a small square of sterile Millipore type HA filter $(0.45 \mu \mathrm{m}$ pore size $)$ submerged in EBSS. Filters were then placed on stainless steel grids in standard organ culture dishes (Falcon 3037). Cultures were fed with a 1:1 mixture of Dulbecco's modified Eagle's minimal essential medium and Ham's nutrient F12 mixture (DMEM/F12, GIBCO, Grand Island, N.Y.), containing $1 \%$ fetal bovine serum, $20 \mu \mathrm{g} / \mathrm{ml}$ gentamicin sulfate, and $2 \%$ B27 culture supplement (GIBCO). Tongue cultures were maintained at the liquid/gas interface $\left(5 \% \mathrm{CO}_{2}\right.$ in air) in a humidified incubator at $37^{\circ} \mathrm{C}$ and the medium was changed daily. After $0,2,3$, or 6 days in culture, tongues were removed, rinsed in phosphate-buffered saline (PBS, $\mathrm{pH}$ 7.4), and either processed for scanning electron microscopy studies or submerged in OCT-compound and rapidly frozen.

Scanning electron microscopy. Tongue cultures were rinsed in PBS, and fixed in 2\% paraformaldehyde and $2.5 \%$ glutaraldehyde sequentially in $0.025 \mathrm{M}$ cacodylate buffer $(\mathrm{pH} 7.3)$ for $4 \mathrm{~h}$ at $4^{\circ} \mathrm{C}$ Cultures were then post-fixed in $1 \%$ aqueous $\mathrm{OsO}_{4}, 1 \%$ tannic acid, and $1 \% \mathrm{OsO}_{4}$ for $1 \mathrm{~h}$ in each solution, and dehydrated in a graded series of ethanol that was exchanged during three subsequent washes in hexamethyldisilazane (HMDS). The HMDS was allowed to evaporate from cultures in a fume hood overnight. Cultures were then mounted on stubs, lightly sputter-coated with gold/palladium, and studied in a scanning electron microscope.

In situ hybridization histochemistry. Serial sections $(14 \mu \mathrm{m})$ of organ cultures were cut on a cryostat and thaw-mounted onto coated slides (ProbeOn, Fisher). Oligonucleotide probes complementary to rat NGF (50-mer probes from bases 398-447; Whittemore et al. 1988), BDNF (bases 250-298; Leibrock et al. 1989), NT-3 (bases 679-726; Maisonpierre et al. 1990) and NT-4 (549-598; Ip et al. 1992) were synthesized (DNA Technology, Aarhus, Denmark). A 50-mer random control probe was also used (Scandinavian Gene Synthesis, Köping, Sweden; Nosrat and Olson 1995). Procedures for in situ hybridization were as described by Dagerlind et al. (1992). Briefly, oligonucleotides were 3'-end labeled with ${ }^{35}$ S-dATP using terminal deoxyribonucleotidyl transferase. Sections were hybridized for $16-20 \mathrm{~h}$ at $42^{\circ} \mathrm{C}$ in a humidified chamber with $3.75 \mu$ labeled probe $\left(=2 \times 10^{5} \mathrm{cpm} / \mu \mathrm{l}\right.$ labeled probe $)$ made to $100 \mu \mathrm{l}$ for each slide in a mixture of $4 \times \mathrm{SSC}(1 \times \mathrm{SSC}=$ $0.15 M \mathrm{NaCl} / 0.015 M$ sodium citrate), $50 \%$ formamide, $1 \times$ Denhardt's solution $(0.02 \%$ each of polyvinyl-pyrrolidone, bovine serum albumin, and Ficoll), $1 \%$ sarcosyl, $0.02 M$ phosphate buffer $(\mathrm{pH} 7.0), 10 \%$ dextran sulfate, $500 \mathrm{mg} / \mathrm{ml}$ heat-denaturated salmon sperm DNA and $200 \mathrm{~m} M$ dithiothreitol. Slides were then 

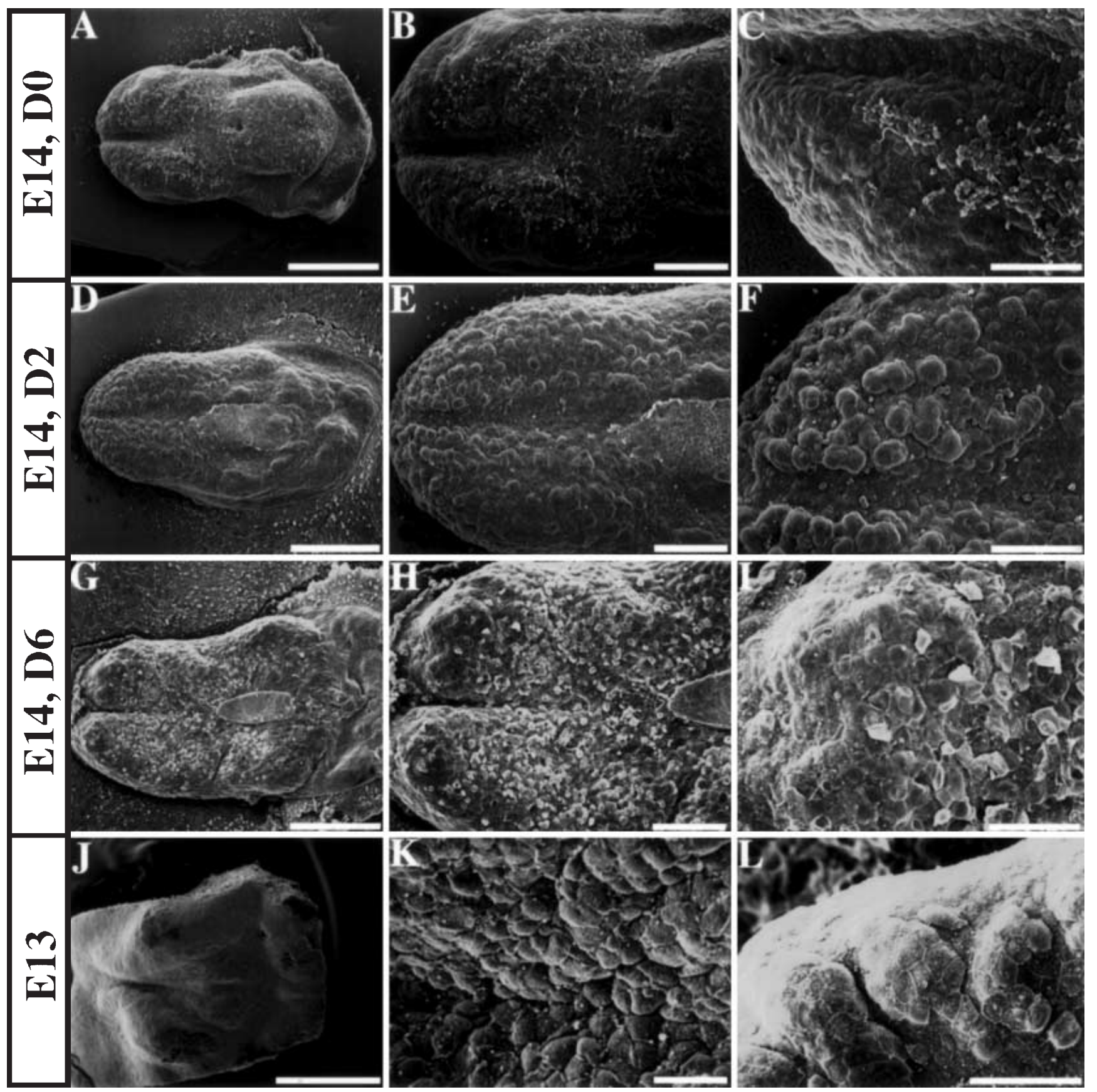

Fig. 1A-L Scanning electron micrographs (SEM) of tongue organ cultures visualizing lingual surface morphology. A-I illustrate tongue cultures started at E14, and $\mathbf{J}-\mathbf{L}$ are cultures started at E13. A-C At E14, on culture day $0(D 0)$, the basic structure of the tongue is apparent, and surface elevations are first emerging that demarcate the developing fungiform papillae (see high magnification of the tongue surface in $\mathbf{C}$ ). D-F After 2 days in culture (D2), fungiform papillae are clearly visible and well elevated above the surrounding tongue surface (see high magnification of the tongue surface in $\mathbf{F}$ ). G-I After 6 days in culture (D6), filiform papillae

rinsed $5 \times 15 \mathrm{~min}$ at $65^{\circ} \mathrm{C}$ in $1 \times \mathrm{SSC}$ and the last SSC rinse was allowed to come to room temperature. Slides were dipped in distilled water, dehydrated through graded series of ethanol $(70 \%$, 95\%, 99.5\%), air dried, dipped in photographic emulsion (Kodak NTB2, diluted 1:1 in distilled water) and exposed for approxi- start to emerge, and fungiform papillae are difficult to distinguish There is also exfoliation of the surface epithelial cells. J, K SEM photomicrographs of the tongue at E13. Note that the lateral swellings that subsequently fuse and form the anterior part of the tongue are observed. $\mathbf{K}$ is higher magnification of the tongue at E13; fungiform papillae are not observed. L After 2 days in culture, the tongue continues to develop, and fungiform papillae are seen as large elevations on the tongue surface. Scale bars represent $500 \mu \mathrm{m}$ in $\mathbf{A}, \mathbf{D}, \mathbf{G}$, and $\mathbf{J}, 200 \mu \mathrm{m}$ in $\mathbf{B}, \mathbf{E}, \mathbf{H}$, and $\mathbf{K}$, and $100 \mu \mathrm{m}$ in $\mathbf{C}, \mathbf{F}$, and $\mathbf{I}$, and $32 \mu \mathrm{m}$ in $\mathbf{L}$

mately 8 weeks. After developing, the slides were counterstained weakly with cresyl violet and mounted (Entellan, Merck, Darmstadt, Germany).

Positive control procedure was based on the characterization of probes for a given mRNA species in our prior studies. Negative 


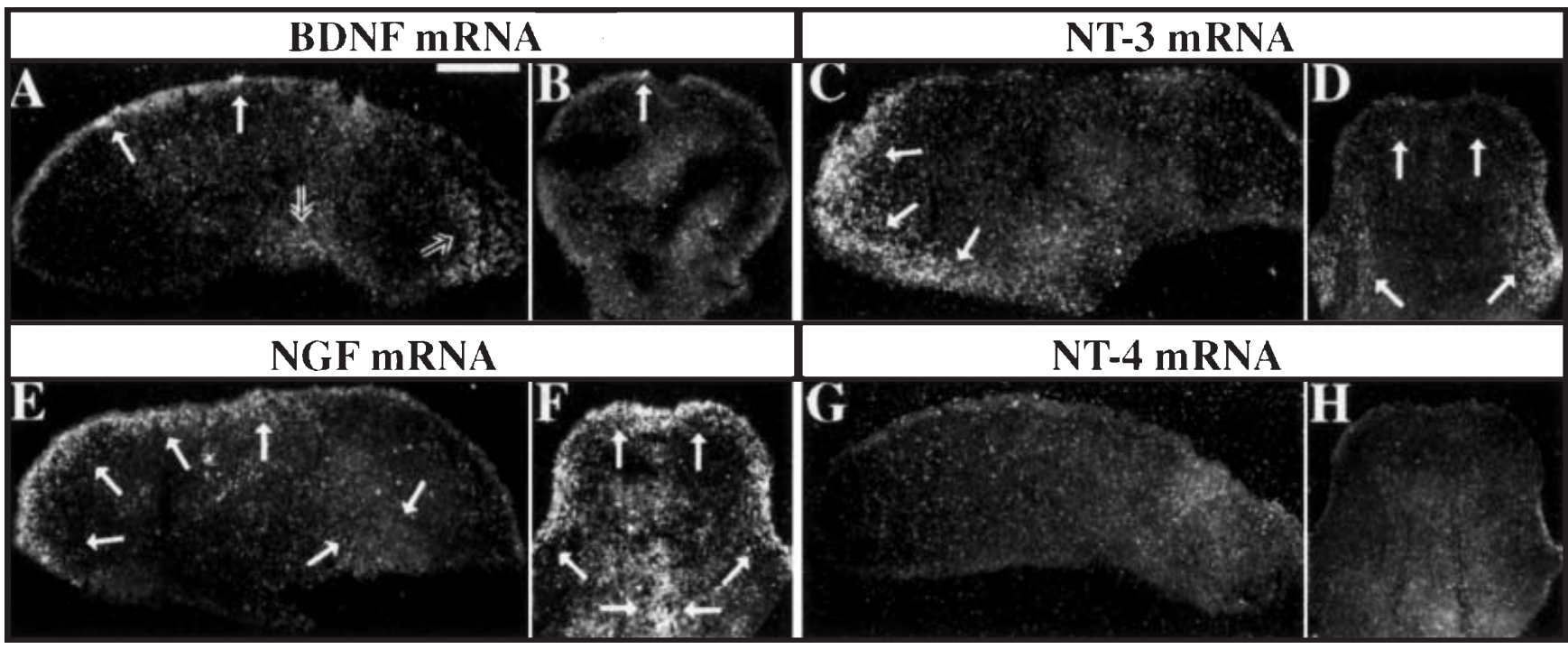

Fig. 2A-H Dark-field photomicrographs, visualizing sagittal and transverse sections of the tongues at E14, and hybridized with oligonucleotide probes specific for different neurotrophin mRNAs. A, B BDNF mRNA labeling is found in small restricted areas in the lingual epithelium (solid arrows) and in the developing tongue muscle tissue (open arrows in A). C, D NT-3 mRNA labeling is found subepithelially, mainly in the anterior parts of the tongue (solid arrows). E, F At E14, NGF mRNA labeling is found subepithelially in the mesenchyme (four solid arrows). The labeling is found for a longer distance posteriorly than that of NT-3 mRNA. In addition, NGF mRNA labeling is also found in the core part of the tongue (two solid arrows pointing towards midline). G, H NT4 mRNA labeling is below the detection level in the lingual epithelium. Scale bar represents $250 \mu \mathrm{m}$

controls were: (1) the inclusion of a random control probe, and (2) different neurotrophin probes functioning as controls for each other since they had similar GC contents. The specificity of the in situ hybridization procedure is dependent on high stringency conditions (in particular rinsing temperature and salt concentration) and the positive and negative controls related above.

Detection of positive autoradiographic signals was based on serial observation of accumulations of silver grains in the emulsion above specific cells and tissues identified by the staining procedures. Only areas over which accumulations were clearly above the surrounding background level, detectable using dark-field microscopy and a primary magnification of $5-10 \times$, were regarded as positive. To allow direct comparisons of distribution of different species of mRNA, serial sectioning and labeling of consecutive sections with different probes were used. All comparisons were thus based on tissues sectioned, hybridized, exposed to emulsion, and further processed together for a given stage. Processing several such pairs gives adequate information and is clearly superior to individual sectioning using one probe at a time. Photomicrographs of the sections were scanned, digitally processed for photoprints with appropriate brightness and contrast, and printed.

\section{Results}

Development of the tongue in vivo

Fungiform papillae were not observed on the dorsal surface of the tongue, when tongue cultures were started either at E14 (Fig. 1A-C), or at E13 (Fig. 1J, K). Tongues cultures starting at E14 continued to grow well in the culture environment, as previously shown (Mbiene et al. 1997), and fungiform papillae developed (better visualized at high magnification; Fig. 1E, F). After 6 days in culture, however, fungiform papillae were not evident (Fig. 1H, I).

The tongue at E13 is still essentially a series of three lingual swellings (Fig. 1J) and the dorsal epithelium is homogeneous, without papillae (Fig. 1K). After 2 days in culture, fungiform papillae were clearly observed on the tongue cultures that were started at E13 (Fig. 1L).

\section{Organ cultures started at embryonic day 14}

Neurotrophin mRNA expression patterns at culture day 0. BDNF transcripts were observed in small restricted elevations of epithelium in the dorsal surface of the tongue at E14, or culture day 0 (solid arrows in Fig. 2A and B). These BDNF-positive elevations correspond to locations of early developing fungiform papillae. BDNF mRNA labeling also was present in tissues at the very base of the tongue and in most posterior lingual regions (open arrows in Fig. 2A). However, BDNF transcripts in the anterior tongue tissue core and/or subepithelial mesenchyme were below levels of detection. NT-3 mRNA labeling was localized in a densely labeled subepithelial band in the anterior half of the tongue (solid arrows in Fig. 2C and D). NT-3 mRNA labeling was, however, below detection among the tongue core of developing muscle tissue. NGF mRNA labeling was similarly distributed in a subepithelial band of mesenchyme in the anterior half of the tongue (Fig. 2E, F). In contrast to NT-3 mRNA labeling, NGF mRNA labeling was also observed among tongue muscle tissue. NT-4 mRNA labeling was below the detection level in the lingual epithelium (Fig. 2G, H).

Neurotrophin mRNA expression patterns in day 2 cultures. Fungiform papillae had formed after 2 days in cul- 


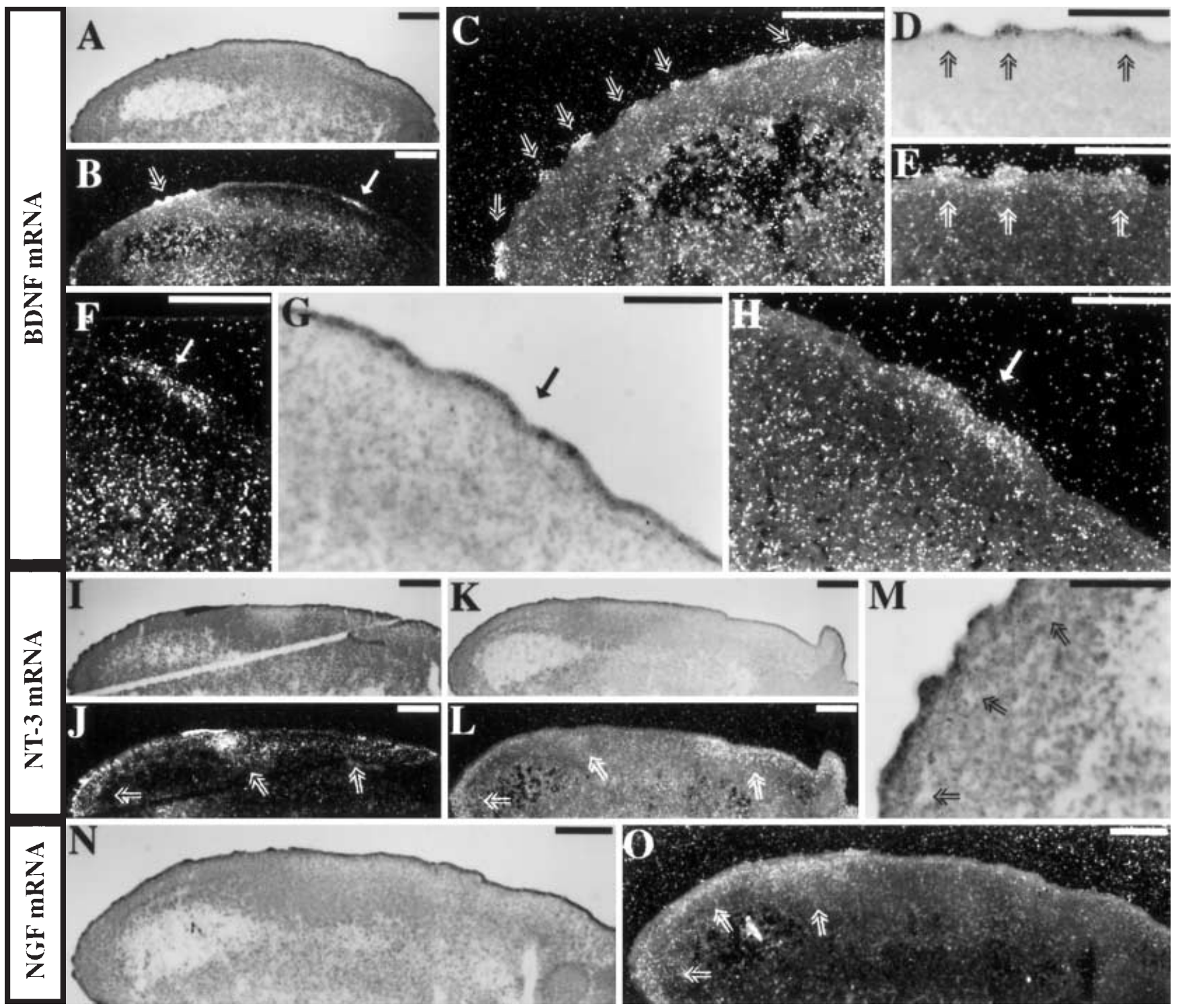

Fig. 3A-O Bright- and dark-field photomicrographs of sagittally sectioned tongue cultures started at E14, and grown in culture for 2 days. Sections were hybridized with specific oligonucleotide probes for different neurotrophin mRNAs. NT-4 mRNA was below detection levels in the tongue and is not shown. The anterior part of the tongue is located to the left in all figures. A, B BDNF mRNA labeling is found in the developing fungiform papillae (open arrow), in the posterior parts of the tongue in the lingual epithelium (solid arrow) representing the developing circumvallate papilla, and in the tongue musculature. $\mathbf{C}$ After 2 days in culture, fungiform papillae are clearly visible and are elevated above the surrounding lingual epithelium. BDNF mRNA labeling is found in the epithelium covering the fungiform papillae (open arrows). D, E Bright- and dark-field photomicrographs of BDNF mRNA labeling in fungiform papillae (open arrows). F Higher magnification of the posterior part of the tongue marked with a solid arrow in B, visualizing the epithelial BDNF mRNA labeling in the area of circumvallate papilla. G, $\mathbf{H}$ Additional photomicrographs, visualizing BDNF mRNA labeling in the epithelium at posterior parts of the tongue, representing the developing circumvallate papilla. I-L In D2 embryonic tongue cultures, NT-3 mRNA labeling (open arrow) is seen subepithelially in the anterior part of the tongue, and in the mesenchyme in the area of the intermolar eminence (middle arrow). At the most posterior part of the tongue, NT-3 mRNA labeling is observed in the mesenchyme as well as in the lingual epithelium (posterior arrow). NT-3 labeling is seen in the mesenchymal core of developing fungiform papillae (best visualized in M). M Higher magnification of NT-3 mRNA labeling (open arrows) in the subepithelial mesenchyme in the anterior part of the tongue. NT-3 mRNA labeling in the lingual epithelium is below the level of detection. $\mathbf{N}, \mathbf{O}$ NGF mRNA labeling is seen subepithelially in the mesenchyme in the anterior parts of the tongue (open arrows). Scale bars represent $250 \mu \mathrm{m}$ in $\mathbf{A}, \mathbf{B}, \mathbf{I}-\mathbf{L}, \mathbf{N}$, and $\mathbf{O}$, and $125 \mu \mathrm{m}$ in $\mathbf{C}-\mathbf{H}$ and $\mathbf{M}$ ture and BDNF mRNA was found in the dorsal surface epithelium covering the developing fungiform papillae (Fig. 3A-E). Occasionally, BDNF mRNA labeling also appeared to be within the interpapilla epithelium in areas of dense papilla development (open arrow in Fig. 3B), but we did not confirm this with serial sections. In the posterior part of the tongue, BDNF mRNA was found in a restricted area in the dorsal surface epithelium (solid 


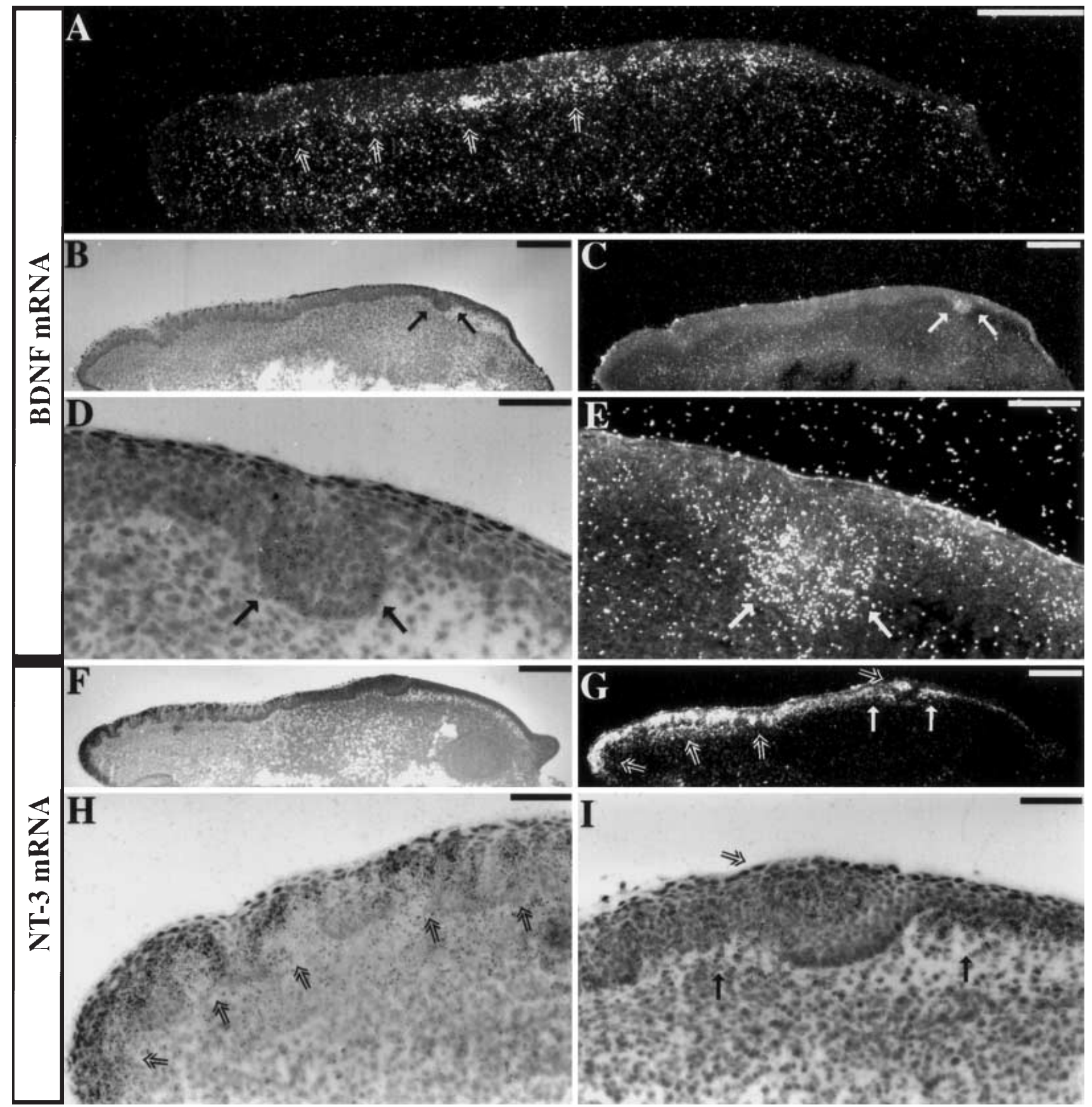

Fig. 4A-I Bright- and dark-field photomicrographs of sagittally sectioned tongue cultures, dissected at E14 and grown in culture for 6 days. Sections were hybridized with specific BDNF and NT-3 oligonucleotide probes. The anterior part of the tongue is located towards the left side in all figures. A BDNF mRNA labeling (open arrows) is diffusely found in the lingual epithelium, and in the core part of the tongue after 6 days in culture. B-E Interestingly, the developing circumvallate trench epithelium contains BDNF mRNA labeling (solid arrows). F, G After 6 days in culture, a strong NT-3 mRNA labeling is observed in the lingual epithelium of the anterior part of the tongue (open arrows). Interestingly, NT-3 mRNA is also observed in the top surface of the developing circumvallate papilla (open arrow in the top part of the photomicrograph). NT-3 mRNA labeling is also observed in the surrounding areas of the papilla (solid arrows), but not in the trench epithelium. H Higher magnification of the anterior part of the tongue in $\mathbf{F}$ and $\mathbf{G}$. NT-3 mRNA labeling is clearly observed in the lingual epithelium, as well as in the underlying mesenchyme. I Higher magnification of the developing circumvallate papilla in $\mathbf{F}$ and G. NT-3 mRNA is also observed in the top surface of the developing circumvallate papilla (open arrow). Trench epithelium is devoid of NT-3 mRNA labeling. Labeling is also found in the surrounding mesenchyme of the papillae (solid arrows). Scale bars represent $250 \mu \mathrm{m}$ in $\mathbf{A}-\mathbf{C}, \mathbf{F}$ and $\mathbf{G}$, and $50 \mu \mathrm{m}$ in $\mathbf{D}, \mathbf{E}, \mathbf{H}$ and $\mathbf{I}$ 

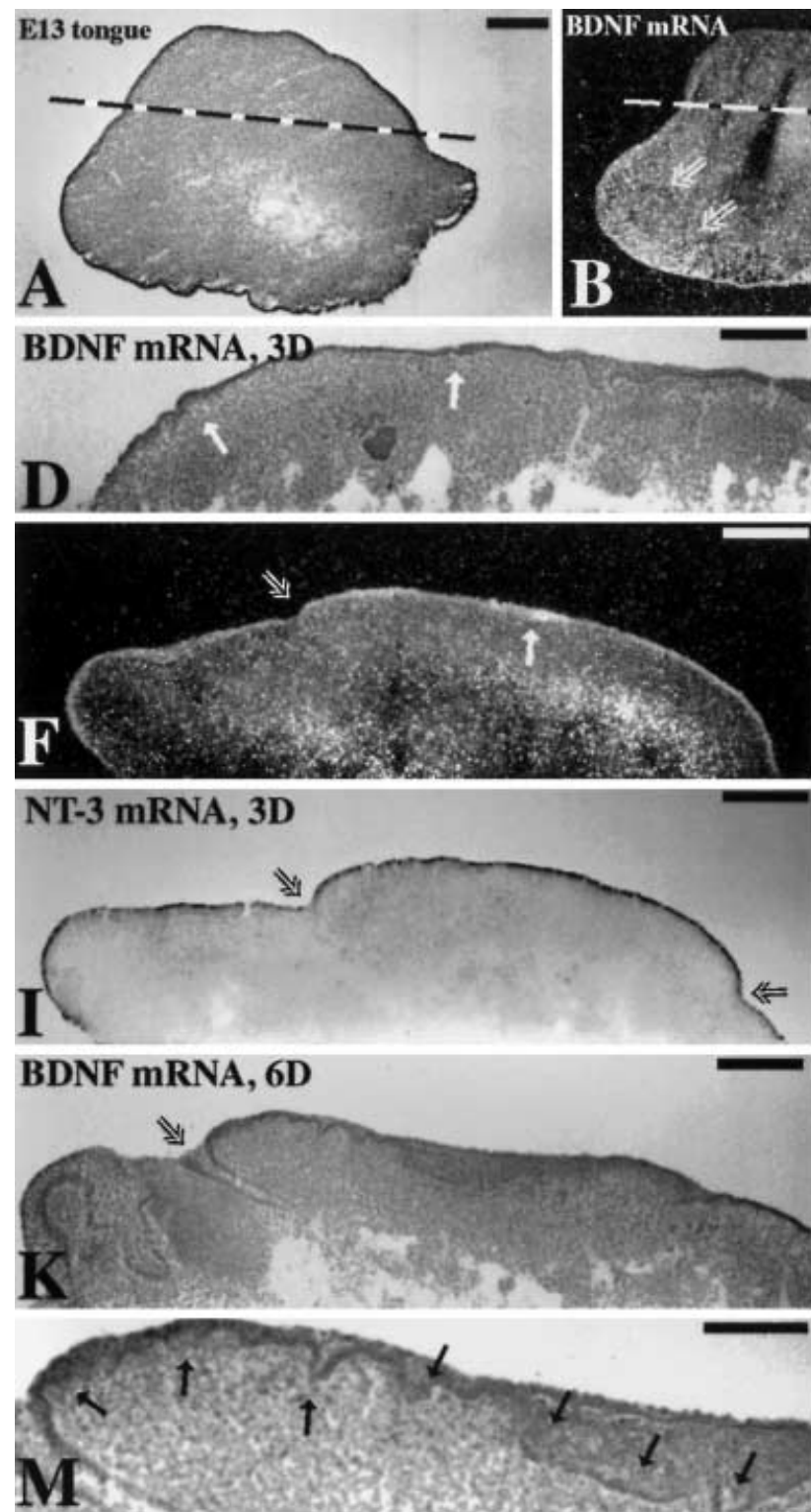

NT-3 mRNA, 6D

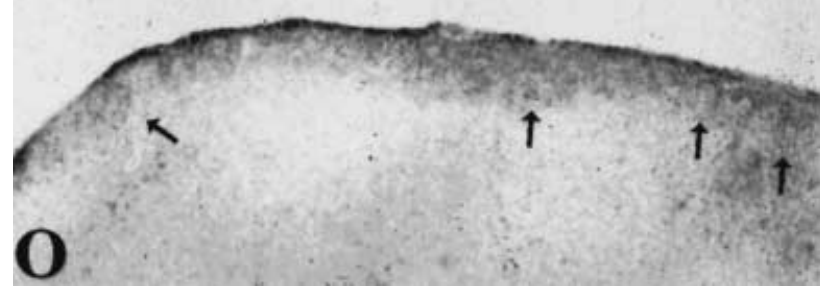

Fig. 5A-P Bright- and dark-field photomicrographs of sagittally sectioned tongue cultures starting at E13 (A-C), and grown in culture for 3 (D-J) or $6(\mathbf{K}-\mathbf{P})$ days. Sections were hybridized with specific BDNF and NT-3 oligonucleotide probes. A-C Sagittal sections of control tongues at E13. B BDNF mRNA labeling is below detection levels in the tongue, but the labeling is found in the anterior part of the mandible (open arrows). C NT-3 mRNA labeling is also found in the anterior part of the mandible (arrows). D, E After 3 days in culture, fungiform papillae develop and BDNF mRNA is localized within the surface epithelium of the papillae (arrows). BDNF mRNA labeling is also observed within the developing muscle tissue (asterisk in $\mathbf{E}$ ). F BDNF mRNA is found in the tongue muscle tissue after 3 days in culture (asterisk), as well as in the lingual epithelium (solid arrow). The open arrow
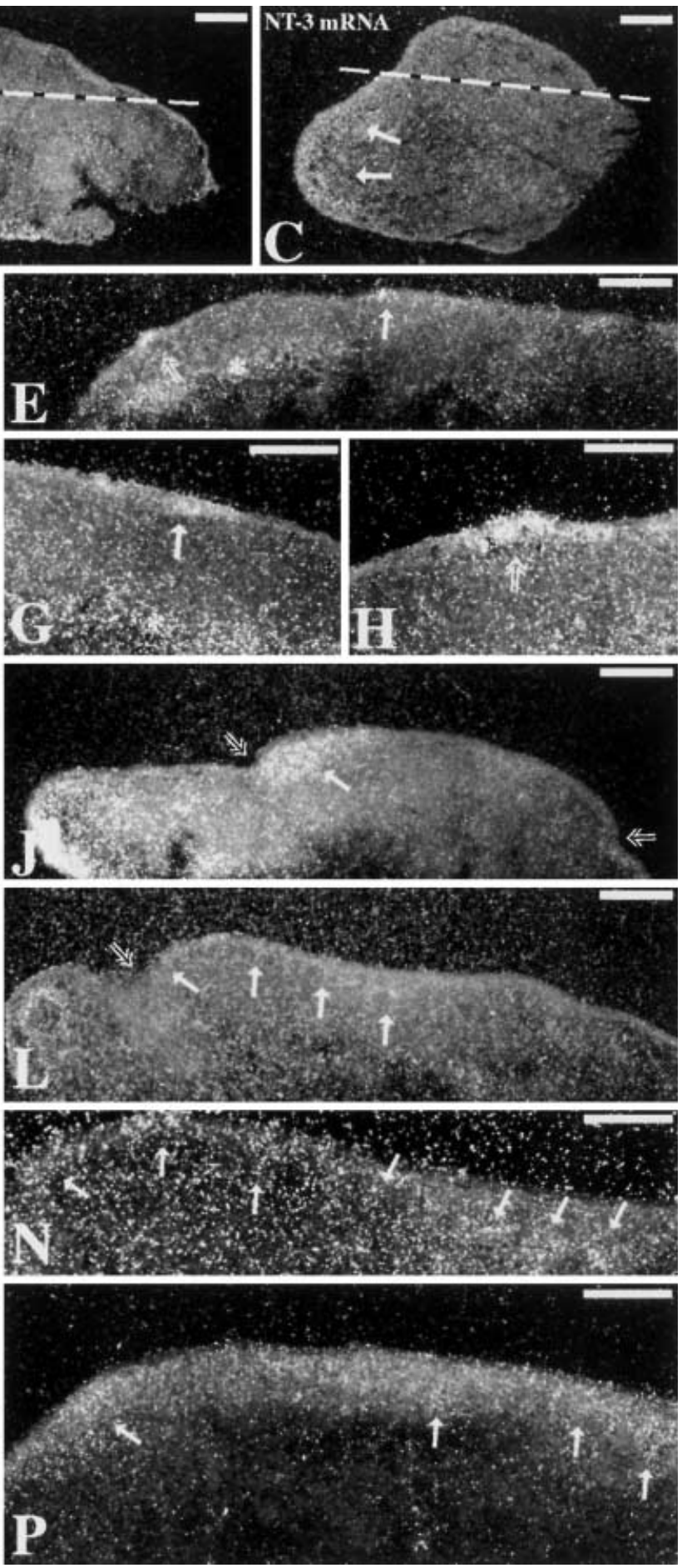

demarcates the anterior border of the tongue. G Higher magnification of the area in $\mathbf{F}$, visualizing the labeling in the lingual epithelium. H Higher magnification of anteriorly located fungiform papilla in E. BDNF mRNA is expressed in the lingual epithelium. I, J After 3 days in culture, NT-3 mRNA labeling is observed in the subepithelial mesenchyme in the anterior part of the tongue (solid arrow). Open arrows demarcate anterior and posterior borders of the tongue. K, L After 6 days in culture, weak BDNF mRNA labeling is observed throughout the lingual epithelium (filled arrows). Open arrow demarcates the anterior border of the tongue. $\mathbf{M}, \mathbf{N}$ Higher magnification of the tongue in $\mathbf{K}$ and $\mathbf{L}$. $\mathbf{O}$, P Weak NT-3 mRNA labeling is observed in the lingual epithelium after 6 days in culture. Scale bars represent $250 \mu \mathrm{m}$ in A-F and $\mathbf{I}-\mathbf{L}$, and $125 \mu \mathrm{m}$ in $\mathbf{G}, \mathbf{H}$, and $\mathbf{M}-\mathbf{P}$ 
arrow in Fig. 3B and Fig. 3F-H) that corresponded to the location of the developing circumvallate papilla. NT-3 mRNA was located subepithelially with a thick bandlike appearance in the tongue organ cultures (Fig. 3I-L). NT-3 labeling was also found in the mesenchymal core of the developing fungiform papillae (best visualized in Fig. 3J and M). In the posterior half of the tongue cultures there was weak labeling in the lingual epithelium as well as in the mesenchyme (Fig. 3J, L). NGF mRNA labeling also was located subepithelially in the day 2 tongue cultures (Fig. 3N, O). The labeling was stronger in the anterior half of the tongue than in the posterior half (open arrows in Fig. 30). Weak labeling was observed among the tongue core of muscle tissue (not visible at this magnification). NT-4 mRNA labeling was below the detection level in the tongue cultures (not shown).

Neurotrophin mRNA expression patterns in day 6 cultures. Distinct fungiform papillae are not clearly retained in the tongue cultures after 6 days (see also Fig. 1J-I), but rather a series of epithelial downgrowths remodel on the anterior tongue in culture that correspond to the emerging filiform papillae (open arrows in Fig. 4A). Developing filiform papillae are seen in the anterior part of the tongue in Fig. 4B. BDNF mRNA was found with a widespread pattern of expression in this type of epithelium in the anterior part of the tongue (open arrows in Fig. 4C). The shift from circumscribed to more widespread BDNF mRNA labeling might be related to the loss of distinct fungiform papilla patterning in 6-day cultures.

An epithelial invagination in the posterior part of the tongue contained BDNF mRNA labeling in the epithelial cells (arrows in Fig. 4B-E). This invagination corresponded to the circumvallate papillae trench in the tongue culture system. As reported previously (Nosrat and Olson 1995), the dorsal surface epithelium of the developing circumvallate papillae is devoid of detectable BDNF mRNA labeling at E19 and onwards in vivo, whereas BDNF labeling is found in the developing trench wall epithelium. Tongues in culture for 6 days correspond to tongues at embryonic day 20 in vivo. Thus BDNF transcripts are found in circumvallate trench epithelium at this stage, both in vivo and in vitro. Weak BDNF mRNA labeling was also observed in the core part of the tongue.

A distinct shift in the expression pattern of NT-3 mRNA labeling was observed in day 6 tongue cultures (Fig. 4F-I). Strong NT-3 labeling was now seen in the lingual epithelium (Fig. 4F-H), as well as in the subepithelial mesenchyme (Fig. 4G, H). Both tissue compartments of filiform papillae, the epithelium and the mesenchymal core, contain NT-3 mRNA labeling (Fig. 4G, H). However, the epithelial labeling appeared stronger than the mesenchymal labeling. NT-3 transcripts were observed in the dorsal surface epithelium of the developing circumvallate papillae (open arrows to the right side of the Fig. 4G and I) but the presumed trench epithelium was devoid of detectable levels of NT-3 transcripts. These patterns of expression correspond well to NT-3 mRNA expression patterns in vivo (Nosrat and Olson 1998). NGF mRNA labeling (not shown) was found in the subepithelial mesenchyme, similar to that of the prior stage (see Fig. 3B) and did not alter in expression pattern. NT-4 mRNA labeling was below detection level.

Neurotrophin mRNA expression patterns in organ cultures started at embryonic day 13

To study the emergence of neurotrophin mRNA expression patterns in cultures begun when the embryonic tongue is still a series of swellings, before papillae have begun to differentiate and when no nerve fibers are found in the proximity of the developing tongue, we initiated tongue cultures at E13. BDNF and NT-3 mRNA expression patterns were examined, because these patterns were most related to epithelial/mesenchymal interactions and papilla development in E14 cultures.

In the E13 tongues (Fig. 5A), at culture day 0, BDNF mRNA labeling was below the detection level in the tongue swellings (Fig. 5B), but labeling was observed in the anterior parts of the developing mandibular process (open arrows in Fig. 5B). NT-3 mRNA labeling also was observed in the anterior part of the mandible (Fig. 5C). Similar results were obtained for both day 2 and day 3 cultures; therefore only day 3 cultures are illustrated. After either 2 or 3 days in culture, fungiform papillae had developed and were elevated above the surrounding lingual epithelium (see also Fig. 1L). BDNF labeling was observed in the epithelium covering the fungiform papillae (arrows in Fig. 5D, E and H), and among the tissue in the core of the tongue (asterisks in Fig. 5E-G). NT-3 mRNA labeling was found subepithelially in the mesenchyme (Fig. 5I, J), and was strongest in mesenchyme of the anterior part of the tongue (solid arrow in Fig. 5J). After 6 days in culture, BDNF mRNA labeling was generally weak and distributed diffusely in the lingual epithelium (solid arrows in Fig. 5L-N). Labeling was also found in the area of the circumvallate papillae trench epithelium (not shown). NT-3 mRNA labeling was generally weak and was found mainly in the epithelium, but also in the underlying subepithelial mesenchyme (Fig. 5O, P). Thus, the NT-3 expression pattern shifted from mesenchymal, to epithelial and mesenchymal, in E13 as well as in E14 cultures.

\section{Discussion}

We studied neurotrophin mRNA expression in tongue organ cultures, thereby excluding influences from sensory nerve fibers on expression patterns. As reported previously (Mbiene et al. 1997), whether tongue cultures were begun at embryonic day 13 or 14, after 2 and 3 days in culture gustatory papillae developed. In the day 2 or day 3 cultures, BDNF mRNA was localized in the gustatory 
epithelium of both the developing fungiform and circumvallate papillae, and NT-3 and NGF mRNA was localized in the subepithelial lingual mesenchyme. These patterns correspond well to those observed during in vivo development (Nosrat and Olson 1995; Nosrat and Olson 1998), and therefore demonstrate retention of appropriate tissue distribution of neurotrophins in embryonic tongue cultures. Furthermore, because the tongue cultures exclude sensory ganglion cells, the experiments demonstrate that neurotrophin expression patterns in taste papillae and tongue tissues emerge independently of sensory nerve regulation.

Although fungiform and circumvallate papillae develop in tongue organ cultures initiated at E13 or E14, after 6 days in culture fungiform papillae are not maintained in the usual pattern on anterior tongue (Mbiene et al. 1997). The fungiform papillae are obscured in the emerging array of filiform papillae that essentially cover anterior tongue or they dedifferentiate. Similarly, BDNF mRNA labeling in tongue cultures is no longer circumscribed but is widespread in anterior tongue epithelium at day 6 . Thus, the fungiform papillae apparently are vulnerable to absence of innervation for sustained morphogenesis and patterning in culture (Mbiene et al. 1997). In contrast, the single circumvallate papilla on the posterior tongue retains at least general papilla form after 6 days in culture (see Fig. 4B-E), and still contains localized BDNF mRNA.

Therefore, we have observed that when papilla morphology is lost in organ cultures (e.g., anterior tongue, fungiform papillae), BDNF mRNA localized expression becomes diffuse. When papillae retain some structure (e.g., posterior tongue, circumvallate papilla), BDNF mRNA expression remains localized to the papilla. We suggest that fungiform papillae and localized BDNF mRNA expression are not retained in 6-day cultures because sensory innervation is required to sustain papilla morphology, once they develop (Mbiene and Mistretta 1997; Mistretta et al. 1999; Morris-Wiman et al. 1999; Nosrat et al. 1997; Zhang et al. 1997). The circumvallate papilla and local BDNF mRNA might be less vulnerable to morphological disruption in the absence of innervation because of the large papilla mass. In vivo, as the trench system of circumvallate papilla is forming, BDNF labeling disappears from the top surface epithelium of the papilla, and moves along with the downgrowing epithelium. At E19 and thereafter, BDNF labeling is found in the trench epithelium in rats. These series of events occur, however, a few days after BDNF labeling is observed in the top surface epithelium of the fungiform papillae in vivo. Therefore, it is also possible that BDNF labeling in the circumvallate trench epithelium in cultured tongues is a result of a developmentally controlled timing for expression of BDNF in the early gustatory epithelium.

Interestingly, the distribution of NT-3 mRNA was altered with time and shifted from solely mesenchymal, to both epithelial and mesenchymal expression in the tongue after 6 days in culture. This replicates in vitro the shift that is observed during in vivo development at about E18 (Nosrat and Olson 1998). Because the organ cultures exclude sensory innervation, the alteration in NT-3 expression pattern is independent of intact innervation. Neurotrophin mRNA expression patterns also have been studied in developing teeth, in vivo and in a culture system (Luukko et al. 1997). As with tongue development, patterns of NT-3 and NGF mRNA expression in vitro resembled those in vivo, indicating that innervation is not required for neurotrophin expression in developing teeth.

The presence of neurotrophins in developing rat tongue epithelium and mesenchyme in vivo is appropriately distributed spatially and temporally to attract and support growing innervation to gustatory and tactile receptors (Nosrat and Olson 1995; Nosrat and Olson 1998). In vitro, BDNF is especially effective in promoting neurite outgrowth from the E16 rat geniculate ganglion, whereas NT-3 and NGF support extensive neurite outgrowth from the trigeminal ganglion (Mistretta 1998; Mistretta et al. 1998). The gustatory ganglia and cranial ganglia related to sensory innervation of the tongue all show neuronal losses in BDNF and NT-3 null-mutated mice (Ernfors et al. 1994a; Ernfors et al. 1994b; Farinas et al. 1994; Jones et al. 1994), again indicating the dependence of these neurons on the survival-promoting actions of specific neurotrophins. Furthermore, in BDNF and NT-3 null mutant mice, selective effects are seen in decreased gustatory and tactile innervation (Mistretta et al. 1999; Nosrat et al. 1997; Zhang et al. 1997). Ectopic overexpression of BDNF in the tongue also leads to gustatory organ deficits (Ringstedt et al. 1999). These data converge to demonstrate a key role for neurotrophins in sustaining tongue and taste organ innervation, which in turn is essential for maintaining developing taste papillae and buds (Mistretta et al. 1999; Nosrat et al. 1997; Zhang et al. 1997).

As sensory nerve fibers grow into the developing tongue, the neurites follow precise and restricted pathways, and various molecules in lingual tissues appear to be involved in growth and branching of the fibers (Mbiene and Mistretta 1997; Mistretta and Haus 1996). Recent data show that these molecules exert not only attractive forces, but also repulsive forces that have been shown to be involved in patterning and distribution of nerve fibers within the tongue (Rochlin and Farbman 1998). Short-range tropic effects of neurotrophins on lingual nerve fibers have also been proposed, and when the neurotrophin BDNF was ectopically overexpressed in the tongue, gustatory fibers did not grow to their correct final targets in the lingual epithelium, but branched among the tongue musculature (Ringstedt et al. 1999). Taken together, it is clear now that many different classes of molecules, exerting both tropic and trophic forces on axons and neurons, are implicated in the establishment of the lingual innervation.

Other regulatory mechanisms that might be operating in neurotrophin mRNA patterning in tongue include epithelial-mesenchymal interactions mediated by Wnt fac- 
tors, recently shown to control NT-3 expression in embryonic limb cultures (Patapoutian et al. 1999). Wnt proteins are important for regulating cell fate, adhesion, and proliferation during development in vertebrate and invertebrate embryos (Cadigan and Nusse 1997). Although this has not been studied in the tongue, it is likely that similar epithelial-mesenchymal interactions would be involved in the regulation of neurotrophin mRNA expression in different tongue compartments. Further, formation of gustatory papillae in a patterned array suggests that protein products of genes that regulate epithelial/mesenchymal interactions in tooth, hair follicle, whisker, and feather formation might also have a formative role in taste organ differentiation (Mistretta 1998). Some relevant candidates have been shown to be present in the developing tongue, for example, sonic hedgehog, bone morphogenic proteins 2 and 4, and fibroblast growth factor 8 (Bitgood and McMahon 1995; Hall et al. 1999a; Jung et al. 1999; Morasso et al. 1995); furthermore, Shh mRNA is expressed in papillae that develop in tongue organ cultures (Hall et al. 1999b).

Our demonstration that localized and timed expression of BDNF, NT-3, and NGF mRNA is retained during development of lingual tissues and taste papillae in tongue cultures reinforces the usefulness of this system for discerning tissue and molecular interactions in taste organ development. Furthermore, BDNF expression in the vallate trench epithelium, and shift of NT-3 expression to lingual epithelium after 6 days in culture, indicates that tissue interactions and signaling events upstream to BDNF and NT-3 expression progress normally in the culture system. The mRNA distributions in embryonic tongue cultures also indicate that neurotrophin expression in lingual tissues is independent of nerves during initial developmental stages. We know that in co-cultures of embryonic tongue and ganglia, fibers from gustatory ganglia grow into the developing tongue and mesenchymal core of taste papillae (Mistretta and MacCallum 1996). Therefore, tongue organ cultures alone, and in co-culture with sensory neurons, can provide systems for our continued testing of hypotheses about roles of nerves, molecules, and local tissues in directing taste papilla and taste bud induction, formation, and maintenance.

Acknowledgements We thank Dr. Irina Nosrat for photographic assistance and suggestions on the manuscript.

\section{References}

Bitgood MJ, McMahon AP (1995) Hedgehog and Bmp genes are coexpressed at many diverse sites of cell-cell interaction in the mouse embryo. Dev Biol 172: 126-138

Cadigan KM, Nusse R (1997) Wnt signaling: a common theme in animal development. Genes Dev 11: 3286-3305

Dagerlind Å, Friberg K, Bean AG, Hökfelt T (1992) Sensitive mRNA detection using unfixed tissue: combined radioactive and non-radioactive in situ hybridization histochemistry. Histochemistry 98: 39-49

Ernfors P, Lee KF, Jaenisch R (1994a) Mice lacking brain-derived neurotrophic factor develop with sensory deficits. Nature 368: $147-150$
Ernfors P, Lee KF, Kucera J, Jaenisch R (1994b) Lack of neurotrophin-3 leads to deficiencies in the peripheral nervous system and loss of limb proprioceptive afferents. Cell 77: 503-512

Farbman AI, Mbiene JP (1991) Early development and innervation of taste bud-bearing papillae on the rat tongue. J Comp Neurol 304: 172-186

Farinas I, Jones KR, Backus C, Wang XY, Reichardt LF (1994) Severe sensory and sympathetic deficits in mice lacking neurotrophin-3. Nature 369: 658-661

Hall JM, Finger TE, MacCallum DK, Mistretta CM (1999b) Sonic hedgehog signaling in rodent tongue cultures. Chem Senses 24

Hall JM, Hooper JE, Finger TE (1999a) Expression of sonic hedgehog, patched and gli1 in developing taste papillae of the mouse. J Comp Neurol 406: 143-155

Ip NY, Ibanez CF, Nye SH, McClain J, Jones PF, Gies DR, Belluscio L, Le BM, Espinosa RD, Squinto SP, Persson H, Yancopoulos GD (1992) Mammalian neurotrophin-4: structure, chromosomal localization, tissue distribution, and receptor specificity. Proc Natl Acad Sci U S A 89: 3060-3064

Jones KR, Farinas I, Backus C, Reichardt LF (1994) Targeted disruption of the BDNF gene perturbs brain and sensory neuron development but not motor neuron development. Cell 76: 989-999

Jung H-S, Oropeza V, Thesleff I (1999) Shh, Bmp-2, Bmp-4 and Fgf- 8 are associated with initiation and patterning of mouse tongue papillae. Mech Dev 81: 203-206

Leibrock J, Lottspeich F, Hohn A, Hofer M, Hengerer B, Masiakowski P, Thoenen H, Barde YA (1989) Molecular cloning and expression of brain-derived neurotrophic factor. Nature 341: 149-152

Luukko K, Arumae U, Karavanov A, Moshnyakov M, Sainio K, Sariola M, Saarma M, Thesleff I (1997) Neurotrophin mRNA expression in the developing tooth suggests multiple roles in innervation and organogenesis. Dev Dyn 210: 117-129

Maisonpierre PC, Belluscio L, Squinto S, Ip NY, Furth ME, Lindsay RM, Yancopoulos GD (1990) Neurotrophin-3: a neurotrophic factor related to NGF and BDNF. Science 247: 1446-1451

Mbiene JP, Mistretta CM (1997) Initial innervation of embryonic rat tongue and developing taste papillae. Acta Anat (Basel) 160: $139-158$

Mbiene JP, MacCallum DK, Mistretta CM (1997) Organ cultures of embryonic rat tongue support tongue and gustatory papilla morphogenesis in vitro without intact sensory ganglia. J Comp Neurol 377: 324-340

Mistretta CM (1972) Topographical and histological study of the developing rat tongue, palate and taste buds. In: Bosma JF (eds) Third symposium on oral sensation and perception: the mouth of the infant. C.C. Thomas, Springfield, Ill, pp 163-187

Mistretta CM (1991) Developmental neurobiology of the taste system. In: Getchell TV (eds) Smell and taste in health and disease. Raven Press, New York, pp 35-64

Mistretta CM (1998) The role of innervation in induction and differentiation of taste organs: introduction and background. Ann N Y Acad Sci 855: 1-13

Mistretta CM, Haus LF (1996) Temporal and spatial patterns of tenascin and laminin immunoreactivity suggest roles for extracellular matrix in development of gustatory papillae and taste buds. J Comp Neurol 364: 535-555

Mistretta CM, MacCallum DK (1996) Neurite outgrowth from sensory ganglia into embryonic rat tongue in a co-culture system. Chem Senses 21: 644

Mistretta CM, Xu Z, MacCallum DK (1998) Neurotrophin effects on neurite extension from cultured explants of embryonic rat geniculate, petrosal and trigeminal ganglia. Chem Senses 23: 603

Mistretta CM, Goosens KA, Farinas I, Reichardt LF (1999) Alterations in size, number, and morphology of gustatory papillae and taste buds in BDNF null mutant mice demonstrate neural dependence of developing taste organs. J Comp Neurol 409: $13-24$

Morasso MI, Mahon KA, Sargent TD (1995) A Xenopus distalless gene in transgenic mice: conserved regulation in distal 
limb epidermis and other sites of epithelial-mesenchymal interaction. Proc Natl Acad Sci U S A 92: 3968-3972

Morris-Wiman J, Basco E, Du Y (1999) The effects of betabungarotoxin on the morphogenesis of taste papillae and taste buds in the mouse. Chem Senses 24: 7-17

Nosrat CA (1998) Neurotrophic factors in the tongue; expression patterns, biological activity, relation to innervation and studies of neurotrophin knockout mice. Annals N Y Acad Sci 855: $28-50$

Nosrat CA, Olson L (1995) Brain-derived neurotrophic factor mRNA is expressed in the developing taste bud-bearing tongue papillae of rat. J Comp Neurol 360: 698-704

Nosrat CA, Olson L (1998) Changes in neurotrophin 3 mRNA expression patterns in the prenatal rat tongue suggest guidance of developing somatosensory nerves to their final targets. Cell Tissue Res 292: 619-623

Nosrat CA, Ebendal T, Olson L (1996) Differential expression of brain-derived neurotrophic factor and neurotrophin 3 mRNAin lingual papillae and taste buds indicates roles in gustatory and somatosensory innervation. J Comp Neurol 376: 587-602

Nosrat CA, Blomlöf J, Elshamy WM, Ernfors P, Olson L (1997) Lingual deficits in BDNF and NT3 mutant mice leading to gustatory and somatosensory disturbances, respectively. Development 124: 1333-1342

Nosrat IV, Lindskog S, Seiger $\AA$, Nosrat CA (2000) Lingual BDNF and NT-3 mRNA expression patterns and their relation to innervation in the human tongue: similarities and differences compared with rodents. J Comp Neurol 417: 133-152

Patapoutian A, Backus C, Kispert A, Reichardt LF (1999) Regulation of neutrotrophin-3 expression by epithelial-mesenchymal interactions: the role of Wnt factors. Science 283: 1180-1183

Ringstedt T, Ibáñez CF, Nosrat CA (1999) Role of BDNF in target invasion in the gustatory system. J Neurosci 19: 3507-3518

Rochlin MW, Farbman AI (1998) Trigeminal ganglion axons are repelled by their presumptive targets. J Neurosci 18: 6840-6852

Whittemore SR, Friedman PL, Larhammar D, Persson H, Gonzales CM, Holets VR (1988) Rat beta-nerve growth factor sequence and site of synthesis in the adult hippocampus. J Neurosci Res 20: 403-410

Zhang CX, Brandemihl A, Lau D, Lawton A, Oakley B (1997) BDNF is required for the normal development of taste neurons in vivo. Neuroreport 8: 1013-1017 